AperTO - Archivio Istituzionale Open Access dell'Università di Torino

Bevacizumab in Combination With Either FOLFOX-4 or XELOX-2 in First-line Treatment of Patients With Metastatic Colorectal Cancer: A Multicenter Randomized Phase II Trial of the Gruppo Oncologico dell'Italia Meridionale (GOIM 2802)

This is a pre print version of the following article:

Original Citation:

Availability:

This version is available http://hdl.handle.net/2318/1770107

since 2021-01-29T19:20:07Z

Published version:

DOI:10.1016/j.clcc.2020.01.003

Terms of use:

Open Access

Anyone can freely access the full text of works made available as "Open Access". Works made available under a Creative Commons license can be used according to the terms and conditions of said license. Use of all other works requires consent of the right holder (author or publisher) if not exempted from copyright protection by the applicable law. 


\section{Bevacizumab in combination with either FOLFOX-4 or XELOX-2 in first-line treatment of patients with metastatic colorectal cancer: A multicenter randomized phase II trial of the Gruppo Oncologico dell'Italia Meridionale (GOIM 2802)}

Evaristo Maiello ${ }^{\mathrm{a}}$, Gabriele Di Maggio ${ }^{\mathrm{a}}$, Stefano Cordio ${ }^{\mathrm{b}}$, Saverio Cinieric ${ }^{\mathrm{c}}$, Francesco Giuliani ${ }^{\mathrm{d}}$, Salvatore Pisconti ${ }^{\mathrm{e}}$, Antonio Rinaldi ${ }^{\mathrm{f}}$, Antonio Febbraro ${ }^{\mathrm{g}}$, Tiziana Pia Latiano ${ }^{\mathrm{a}}$, Michele Aieta $^{\mathrm{h}}$, Antonio Rossi ${ }^{\mathrm{a}}$, Daniele Rizzi ${ }^{\mathrm{i}}$, Massimo Di Maio ${ }^{1, \mathrm{~m}}$, Giuseppe Colucci ${ }^{\mathrm{i}}$, Roberto Bordonaro ${ }^{\mathrm{b}}$

aMedical Oncology Unit, Fondazione IRCCS “Casa Sollievo della Sofferenza”, San Giovanni Rotondo (Foggia), Italy

${ }^{\mathrm{b}}$ Medical Oncology Unit, Ospedale Garibaldi, Catania, Italy

${ }^{\mathrm{c}}$ Medical Oncology Unit, Antonio Perrino Hospital, Brindisi, Italy

${ }^{\mathrm{d}}$ Medical Oncology Unit, National Cancer Institute IRCCS "Giovanni Paolo II", Bari, Italy

${ }^{\mathrm{e} O n c o l o g y}$ Unit, SG Moscati Hospital of Taranto, Taranto, Italy

${ }^{\text {f}}$ Ospedale Civile, Castellaneta, (TA), Italy

goncology Unit, Ospedale Sacro Cuore di Gesù, Fatebenefratelli, Benevento, Italy

${ }^{\mathrm{h}}$ Medical Oncology Unit, Department of Onco-Hematology, IRCCS-CROB, Referral Cancer Center of Basilicata, Rionero in Vulture (PZ), Italy

${ }^{i}$ Gruppo Oncologico Italia Meridionale (GOIM) Trial Office, Bari, Italy

${ }^{\text {lDepartment }}$ of Oncology, University of Turin, Turin, Italy

medical Oncology Unit, “Ordine Mauriziano” Hospital, Turin, Italy 
CORRESPONDENCE TO: Evaristo Maiello, MD

Division of Medical Oncology,

Fondazione IRCCS Casa Sollievo della Sofferenza,

Viale Padre Pio, 1

71013, San Giovanni Rotondo (FG), Italy

Phone: +390882410716

Fax: +390882 204095

Email: e.maiello@libero.it

\section{RUNNING TITLE: Bevacizumab plus FOLFOX-4 or XELOX-2 in mCRC patients}

\section{THIS WORK WAS PRESENTED IN PART AT:}

The $54^{\text {th }}$ Annual Meeting of the American Society of Clinical Oncology, Chicago, IL, June 1-5, 2018 [Gastrointestinal (Colorectal) Cancer - Poster Session, abstract 3452].

\section{DisCLAIMERS}

GOIM 2802 is a non-profit trial. The Gruppo Oncologico Italia Meridionale (GOIM) was the sponsor of the trial and was responsible for study design, trial performing, data collection and analysis, report writing and decision to submit for publication.

GOIM 2802 was available in public registries with the following codes: EudraCT number 2010022091-31 


\section{Micro-AbSTRACT}

Bevacizumab plus either oxaliplatin, folinic acid and infusional 5-fluorouracil (FOLFOX-4) or capecitabine plus oxaliplatin (XELOX-2) is among standard first-line treatment options in metastatic colorectal cancer $(\mathrm{mCRC})$ patients. This phase II randomized non-comparative trial evaluated the first-line combination of bevacizumab with either FOLFOX-4 or biweekly XELOX-2 in mCRC patients. Comparable response and a better tolerability for bevacizumab plus XELOX-2 was reported. 


\begin{abstract}
Introduction: Biweekly schedule of capecitabine plus oxaliplatin (XELOX-2) showed interesting results in first-line therapy of metastatic colorectal cancer (mCRC) patients. Bevacizumab plus oxaliplatin, folinic acid and infusional 5-fluorouracil (FOLFOX-4) is among standard first-line treatment options in this setting. We performed a phase II randomized trial in order to evaluate the activity of bevacizumab plus either FOLFOX-4 or XELOX-2 in first-line therapy of mCRC patients.
\end{abstract}

Materials and Methods: $\mathrm{mCRC}$ patients were randomized, in a 1:2 ratio, to first-line bevacizumab plus either FOLFOX-4 (Arm A), as calibration arm, or XELOX-2 (Arm B), up to 12 cycles. Patients without progression were further randomized to maintenance bevacizumab alone or with the same induction fluoropyrimidine. Primary endpoint was objective response rate (ORR), secondary endpoints included progression-free survival (PFS), overall survival (OS) and toxicity. Study design was formally non-comparative, but exploratory comparison was performed.

Results: 45 patients were randomized in arm A and 87 in arm B with an ORR of $55.6 \%$ vs. $48.3 \%$ $(p=0.43)$, respectively. After a median follow-up of 47.2 months, PFS was 10.0 vs. 9.9 months (hazard ratio $[\mathrm{HR}] 0.96,95 \%$ confidence interval [CI] 0.65-1.41; $\mathrm{p}=0.84$ ) and OS was 29.8 vs. 25.0 months (HR 1.21, 95\% CI 0.77-1.92; $\mathrm{p}=0.41)$, respectively. The main grade $3-4$ toxicities $(\%$ A/B) were: neutropenia 15/3, nausea $9 / 5$.

Conclusion: This exploratory analysis showed that biweekly XELOX-2 plus bevacizumab has comparable ORR as FOLFOX-4 plus bevacizumab in patients with mCRC.

KEYWORDS: capecitabine, elderly, fluoropyrimidine, frail, non-comparative 


\section{INTRODUCTION}

The new diagnoses of colorectal cancer (CRC) are nearly 1.4 million every year worldwide, and its incidence is increasing year-by-year. ${ }^{1}$ About $25 \%$ of patients have metastatic disease (mCRC) at diagnosis, and approximately $50 \%$ of patients who have undergone surgery for early stages with curative intent will eventually develop mCRC. ${ }^{2}$

In mCRC patients, systemic therapy is the standard-of-care with a global 25- to 30-month median overall survival (OS), which has been constantly increasing in the last decades. Along with leadtime bias, better and more sophisticated surgery for liver metastases and better support at the end of life, the progress can be reasonably attributed to the introduction in clinical practice of several cytotoxic and targeted agents. ${ }^{3}$ With the exception of patients eligible for a 3 -cytotoxic drugs combination, the typical first-line chemotherapy backbone for fit patients comprises a fluoropyrimidine (intravenous 5-fluorouracil [5-FU] or oral capecitabine) used in various combinations and schedules with irinotecan (FOLFIRI, XELIRI) or oxaliplatin (FOLFOX, XELOX). Cetuximab and panitumumab, anti-EGFR (epidermal growth factor receptor) antibodies $(\mathrm{mAb})$, are widely used in combination with chemotherapy, but limited to patients whose tumors is $R A S$ wild type. Capecitabine-based therapy is not recommended in combination with EGFR mAb. Bevacizumab, a mAb binding circulating VEGF-A (vascular endothelial growth factor-A), in combination with 5-FU/leucovorin/irinotecan or fluoropyrimidine plus oxaliplatin or in combination with 5-FU/leucovorin or capecitabine alone is among standard first-line treatment options, regardless $R A S$ mutational status. Maintenance therapy, with the combination of a fluoropyrimidine plus bevacizumab, is also recommended after the induction therapy with FOLFOX or XELOX. ${ }^{4}$

A phase III study compared FOLFIRI versus FOLFOX-4, in first-line setting of mCRC patients, showing superimposable efficacy results with a different safety profile, which was mild in both arms. ${ }^{5}$ The search for alternative schedules, to optimize the efficacy/tolerability ratio, and uniform the cycle timing of the different regimens, remain a topic of current interest. In a phase II trial, 
biweekly XELOX showed activity and safety profiles similar to those of standard triweekly XELOX. ${ }^{6}$ In the same setting of patients, an our previous phase II study demonstrated a good activity and tolerability of a biweekly schedule of capecitabine combined with oxaliplatin $(\mathrm{XELOX}-2){ }^{7}$

Based on these considerations, we designed this randomized phase II trial in order to evaluate the activity of XELOX-2 plus bevacizumab with FOLFOX-4 plus bevacizumab as calibration arm in first-line therapy of mCRC patients.

\section{MATERIALS AND METHODS}

\section{Eligibility criteria}

Eligible patients had previously untreated histological diagnosis of unresectable mCRC, measurable disease according to the Response Evaluation Criteria in Solid Tumors (RECIST), version 1.1, Eastern Cooperative Oncology Group (ECOG) performance status (PS) 0 or 1, and age 18-75 years, life expectancy $\geq 3$ months, adequate organ function, signed informed consent. The determination of the KRAS mutational status was required, too. When this study was planned and at the beginning of conduction, the "all $R A S$ " determination was not routinely required for clinical practice.

Key exclusion criteria were the presence of any unstable systemic disease or medical contraindication to the study medications, other malignancies within 5 years (except for adequately treated carcinoma in situ of the cervix or basal or squamous cell skin cancer or surgically resected), presence of brain metastases.

The protocol was approved by Institutional Ethical Committees at each participating centre.

\section{Treatment and trial procedures}

The GOIM 2802 study is a phase II randomized non-comparative trial in which mCRC patients were randomized to receive, in a 1:2 ratio, bevacizumab, administered as a 30 - to 90-minute 
intravenous infusion before oxaliplatin at a dose of $5 \mathrm{mg} / \mathrm{kg}$ on day 1 , plus FOLFOX-4 or XELOX2. FOLFOX-4 regimen, the calibration arm (A), included oxaliplatin $85 \mathrm{mg} / \mathrm{m}^{2}$ on day 1 plus folinic acid $100 \mathrm{mg} / \mathrm{m}^{2}$ administered as a 2-hour infusion before $5-\mathrm{FU} 400 \mathrm{mg} / \mathrm{m}^{2}$ administered as an intravenous bolus injection, and 5-FU $600 \mathrm{mg} / \mathrm{m}^{2}$ as a 22-hour infusion immediately after FU bolus injection on days 1 and 2. The XELOX-2 experimental arm (B) included 2-hour intravenous infusion of oxaliplatin $100 \mathrm{mg} / \mathrm{m}^{2}$ on day 1 followed by oral capecitabine $1,000 \mathrm{mg} / \mathrm{m}^{2}$ twice daily on day 1 through 7. Both arms were recycled every 2 weeks for a total of 12 cycles (induction phase). Patients who did not progress at the completion of the induction phase of the trial were further randomized to maintenance with bevacizumab alone or in combination with fluoropyrimidine (capecitabine or 5-FU) (Figure 1).

Dose reductions and delays of chemotherapy due to toxicity were applied as in clinical practice. The use of granulocyte-colony-stimulating factors (G-CSF) was allowed as secondary prophylaxis, in case of grade 4 neutropenia while it was contraindicated during capecitabine administration.

Patient assignment was performed centrally by a phone call to the coordinating center.

\section{Outcomes}

The primary endpoint was the objective response rate (ORR) assessed by Investigators according to RECIST 1.1. According to the intention-to-treat principle, patients not evaluated because of death or toxicity or refusal or loss to follow-up prior to the first restaging, were conservatively considered as non-responders.

Secondary endpoints were progression-free survival (PFS), OS and toxicity. PFS was defined as the time between the date of randomization and the date of disease progression or death, whichever occurred first. Patients who were alive without progression were censored on the date of the last follow-up visit. OS was defined as the time between the date of randomization and the date of death or the date of last follow-up visit. 
Adverse events were coded according to Common Terminology Criteria for Adverse Events CTCAE version 4.0 and toxicity was described as the worst grade suffered for each item by each patient at any time during the treatment.

\section{Statistical analysis}

Sample size of experimental arm was calculated according to Simon's two-stage design, with a type I error rate 0.05 and 0.90 power. With null hypothesis ORR $32 \%$ and alternative hypothesis ORR $48 \%, 46$ patients had to be accrued in the first stage, for a final number of 80 patients. Sample size was increased by $10 \%$ to 88 patients in experimental arm, and 132 patients in the whole study. Study design was formally non-comparative, but exploratory comparison between arms was performed.

Analyses of activity and efficacy were performed on the basis of intention to treat principle. The significance of the difference in the proportion of patients who had an ORR between study treatments groups were compared, with an exploratory aim, by the chi square test. Median followup was calculated according to the reverse Kaplan-Meier technique. ${ }^{8}$ PFS and OS were calculated according to the Kaplan-Meier product-limit method, and curves were compared, with an exploratory aim, with the log-rank test.

All patients who received treatment at least once were included in toxicity analysis. The worst toxicity grade was calculated for each patient.

\section{RESULTS}

\section{Patient characteristics}

Between June 2011 and October 2015, 132 patients were randomized in eight Italian centers (Table 1-Appendix). Overall, 45 patients were assigned to receive FOLFOX-4 plus bevacizumab and 87 to receive XELOX-2 plus bevacizumab (Figure 1). All patients were included in all outcomes analyses. Baseline characteristics of the patients were balanced between the arms and exon $2 K R A S$ 
mutation was detected in $64 \%$ of patients enrolled in arm A and $69 \%$ of cases in arm B (Table 1). Twenty-two patients in arm A, after the completion of 12 cycles of induction treatment, were randomized to the maintenance phase of the study to receive bevacizumab plus 5-FU (arm C: 11 patients) or bevacizumab alone (arm D: 11 patients). Similarly, after the completion of 12 cycles of induction treatment, 47 patients in arm B were randomized to bevacizumab plus capecitabine (arm E: 24 patients) or bevacizumab alone (arm F: 23 patients). All patients were included in the outcomes analyses (Figure 1).

\section{Treatment compliance}

In arm A the median number of treatment cycles was 12 (interquartile range [IQR] 9-19) and in arm B, too (median 12; IQR 9-19). Overall, 27 patients treated in arm A and 55 patients treated in arm $\mathrm{B}$, completed the planned 12 cycles of induction treatment. Five patients in arm A and eight patients in arm B stopped treatment due to patient decision, physician decision or unacceptable toxicity.

\section{Primary endpoint}

Data from the GOIM 2802 trial were locked on September 2017. The ORR was reached in 25 $(55.6 \%)$ patients in arm A versus 42 patients $(48.3 \%)$ in arm B $(\mathrm{p}=0.43)$. A complete response was reported in two (4.4\%) and three (3.4\%) patients, respectively. The disease control rate (DCR) was reached in $39(86.7 \%)$ and $80(92.0 \%)$ patients, respectively (Table 2). No further responses were reported in the maintenance phase of the study.

\section{Secondary endpoints}

With a median follow-up of 47.2 months, events for PFS analyses were 115 (39 in arm A and 76 in arm B). Median PFS was 10.0 in arm A versus 9.9 months in arm B (hazard ratio [HR] 0.96, 95\% confidence interval [CI] 0.65-1.41; $\mathrm{p}=0.84$ ) (Figure 2A). Overall, 79 deaths were recorded (30 in 
$\operatorname{arm~A}$ and 49 in arm B) with a median OS of 29.8 and 25.0 months (HR 1.21, 95\% CI 0.77-1.92; p $=0.41)$, respectively (Figure 2B).

The main grade 3-4 toxicity rate (A versus B) were as follows: thrombocytopenia 2/2, anemia 4/3, neutropenia $15 / 3$, nausea $9 / 5$, vomiting $2 / 3$, diarrhea $7 / 7$, neurotoxicity $2 / 2$ and hypertension $2 / 1$ (Table 3). The toxicity of the maintenance phase was mild (Tables 2 and 3-Appendix).

The KRAS-mutated subgroup analyses showed no differences in any outcomes between the two arms (Table 4-Appendix; Figure 1A and B-Appendix).

\section{DisCUSSION}

The GOIM 2802 study showed that the biweekly bevacizumab plus XELOX-2 regimen is active and well tolerated as first-line therapy of mCRC patients. The study was not designed to perform a formal comparison with the standard bevacizumab plus FOLFOX-4 schedule, which was used as calibration arm, but the outcome of patients treated with the two different treatment strategies was comparable. The ORR reached by the experimental arm was $48.3 \%$ with $43.7 \%$ of patients remaining stable disease. A median PFS of 9.9 months and a median OS of 25 months were reached. Furthermore, bevacizumab plus XELOX-2 regimen showed a manageable safety profile with a good hematological toxicity pattern.

Before the conduction of this trial, the biweekly XELOX schedule was investigated in two phase II studies. $^{7,9}$ A phase II study enrolled 59 patients showing an ORR of $51 \%$ and a DCR of $76 \%$. The preliminary median time to progression (TTP) was 6 months. The treatment was well tolerated with a grade 4 diarrhea reported in only one patient. ${ }^{7}$ Another phase II trial enrolled 35 elderly patients (age $\geq 70$ years) with an ORR of $49 \%$ and the DCR of $86 \%$. Median TTP and OS were 8.6 and 15.5 months, respectively. Grade 3 toxicities were reported in $17 \%$ of patients. ${ }^{9}$

The addition of bevacizumab to the biweekly XELOX was investigated in several trials. ${ }^{10-12}$ In the ORION phase II randomized trial, the biweekly XELOX plus bevacizumab regimen was compared to the triweekly XELOX plus bevacizumab schedule as salvage therapy for 46 patients in whom 
reintroduction of oxaliplatin had been planned as a third- or later-line regimen. There were no differences in any outcome but the safety profile was in favor of the biweekly versus the triweekly regimen with grade $3-4$ fatigue of $21.7 \%$ versus $27.3 \%$, neuropathy and diarrhea $0 \%$ versus $9.1 \%$, respectively. ${ }^{10}$ The PHOENiX Japanese trial, ${ }^{11}$ enrolled 51 untreated patients to receive biweekly bevacizumab plus XELOX regimen reporting an ORR of 51\% with a median PFS of 11.3 months. The grade 3-4 neutropenia, peripheral neuropathy, and hypertension were reported in $13.7 \%$ of patients. A phase II randomized trial enrolled 435 untreated mCRC patients to receive triweekly versus biweekly XELOX regimen plus bevacizumab. The median PFS was 9.6 versus 9.1 months with a median OS of 28.4 versus 22.1 months, respectively. Thus, the triweekly XELOX plus bevacizumab regimen remained the preferred schedule. ${ }^{12}$

In all these trials, ${ }^{9-12}$ the dose of drugs of the biweekly XELOX regimen were slight different from those used in the present trial except for the bevacizumab dose which was the standard $5 \mathrm{mk} / \mathrm{kg}$ every 2 weeks. In fact, the oxaliplatin dose was $85 \mathrm{mg} / \mathrm{m}^{2}$ which was lower than the dose we used in the present study $\left(100 \mathrm{mg} / \mathrm{m}^{2}\right)$. Oxaliplatin at the dose of $100 \mathrm{mg} / \mathrm{m}^{2}$ was defined in order to maintain almost the same dose-intensity of the triweekly regimen $\left(130 \mathrm{mg} / \mathrm{m}^{2}\right)$. Some studies $\mathrm{s}^{9,10}$ used the dose of capecitabine of $1,000 \mathrm{mg} / \mathrm{m}^{2}$ twice daily like in our study while in other trials ${ }^{11,12}$ the capecitabine dose was $\geq 1,500 \mathrm{mg} / \mathrm{m}^{2}$ twice daily. Although the drugs dose-intensity was slight different between these studies, there was no impact on the activity of the biweekly XELOX plus bevacizumab regimen but a slight worsening of hand-foot syndrome toxicity was reported by the trials employing higher dose of capecitabine. ${ }^{11,12}$ The modified regimen used in the current study was well tolerated and relatively easy to administer, as demonstrated by the relatively high median relative dose intensity for both capecitabine and oxaliplatin.

No grade 4-5 toxicities were detected. This favorable safety profile could lead to a reduced need for medications to manage adverse events.

In the present study, the determination of the KRAS status was required. In fact, at the time of the study design, the determination of NRAS status was not mandatory. The results of the KRAS- 
mutated patients were superimposable to those of the overall population. All the other trials ${ }^{9-12}$ did not report any information about this subgroup of patients. This might be of interest also to understand whether subgroup of patients might not benefit of this biweekly schedule.

The GOIM 2802 trial included also the maintenance phase in which patients who completed the induction phase were randomized to bevacizumab alone or in combination with fluoropyrimidine (capecitabine or 5-FU). At the time when this study was designed and started no clear data were available about the best maintenance approach: bevacizumab alone or in combination with fluoropyrimidine. However, the small number of patients participating to the maintenance phase of the study further limit any potential conclusion. Furthermore, the maintenance part of this trial could be responsible of a potential dilution of PFS which was balanced anyway between the arms due to the second randomization. Moreover, this study phase was only explorative and more deeply analyses are ongoing and will be part of future publication.

Our study has several limitations. It was a randomized phase II non-comparative trial. However, we reported the comparisons because the standard arm was considered as a calibration group increasing the strength of the results showed in the experimental arm.

The accrual was very slow, but 94 (71\%) out of the 132 randomized patients were enrolled in two centers. It means that the most of patients were enrolled consecutively with a low potential impact on the enrollment of high selected patients. Moreover, the outcomes achieved with the adapted regimen used in our series is in line with those reported in the above-mentioned trials.

The median age of enrolled patients was 65 years, which was similar to the other trials but one ${ }^{12}$ in which it was 60 years. A study was specifically addressed to elderly patients ${ }^{9}$ underlining that this biweekly schedule might be considered specifically in this group of patients, who generally have several comorbidities receiving more concomitant medications than younger ones also allowing a more exhaustive clinical control, particularly in terms of toxicity.

\section{COnClusions}


The GOIM 2802 exploratory analysis trial confirmed that the biweekly XELOX-2 plus bevacizumab regimen is active and well tolerated. Given the tolerability and convenience of administration, this regimen might be particularly suitable for the treatment of frail or elderly patients. Further trials in this setting are warranted. 


\section{Clinical Practice Points}

- The new diagnoses of colorectal cancer (CRC) are nearly 1.4 million every year worldwide, and its incidence is increasing year-by-year. About $25 \%$ of patients have metastatic disease (mCRC) at diagnosis, and approximately $50 \%$ of patients who have undergone surgery for early stages with curative intent will eventually develop mCRC.

- In mCRC patients, systemic therapy, including a fluoropyrimidine in combination with irinotecan or oxaliplatin with or without cetuzimab or panitumumab or bevacizumab, is the standard-of-care with a global 25- to 30-month median overall survival (OS), which has been constantly increasing in the last decades.

- Biweekly schedule of capecitabine plus oxaliplatin (XELOX-2) showed interesting results in first-line therapy of mCRC patients. Bevacizumab plus oxaliplatin, folinic acid and infusional 5fluorouracil (FOLFOX-4) is among standard first-line treatment options in this setting. We performed a phase II randomized trial in order to evaluate the activity of bevacizumab plus either XELOX-2 or FOLFOX-4 in first-line therapy of mCRC patients.

- Despite the study design was formally non-comparative, an exploratory comparison was performed. The biweekly XELOX-2 plus bevacizumab showed comparable objective response rate as FOLFOX-4 plus bevacizumab with a better tolerability in patients with mCRC.

- Given the tolerability and convenience of administration, bevacizumab plus XELOX-2 might be particularly suitable for the treatment of frail or elderly patients. 


\section{LEGEND TO FIGURES}

Figure 1: Study flow according to CONSORT (FOLFOX-4: oxaliplatin plus folinic acid plus 5fluorouracil [5-FU]; XELOX-2: oxaliplatin plus capecitabine; PD: progressive disease)

Figure 2: Progression-free survival (panel A); Overall survival (panel B)

Figure 1 (Appendix): Progression-free survival (panel A); Overall survival (panel B) in mutated $K R A S$ subgroup 


\section{ACKNOWLEDGMENTS}

The authors thank all the patients who participated in the study; We thank Roche S.p.A. Italy for unconditional contribution. 


\section{REFERENCES}

1. Ferlay J, Soerjomataram I, Dikshit R, Eser S, Mathers C, Rebelo M, et al. Cancer incidence and mortality worldwide: sources, methods and major patterns in GLOBOCAN 2012. Int J Cancer. 2015;136(5):E359-386.

2. Cunningham DL, Atkin W, Lenz HJ, Lynch HT, Minsky B, Nordlinger B, et al. Colorectal cancer. Lancet. 2010;375(9719):1030-1047.

3. Jawed I, Wilkerson J, Prasad V, Duffy AG, Fojo T. Colorectal cancer survival gains and novel treatment regimens: A systematic review and analysis. JAMA Oncol. 2015;1(6):787-795.

4. Van Cutsem E, Cervantes A, Adam R, Sobrero A, Van Krieken JH, Aderka D, et al. ESMO consensus guidelines for the management of patients with metastatic colorectal cancer. Ann Oncol. 2016;27(8):1386-1422.

5. Colucci G1, Gebbia V, Paoletti G, Giuliani F, Caruso M, Gebbia N, et al. Phase III randomized trial of FOLFIRI versus FOLFOX4 in the treatment of advanced colorectal cancer: a multicenter study of the Gruppo Oncologico Dell'Italia Meridionale. J Clin Oncol. 2005;23(22):4866-4875.

6. Scheithauer W1, Kornek GV, Raderer M, Schüll B, Schmid K, Kovats E, et al. Randomized multicenter phase II trial of two different schedules of capecitabine plus oxaliplatin as first-line treatment in advanced colorectal cancer. J Clin Oncol. 2003;21(7):1307-1312.

7. Di Maggio G, Leo S, Nanni L, Giuliani F, Sponziello F, Biglietto M, et al. Xelox-2 (bi-weekly administration of capecitabine + oxaliplatin) as first-line therapy of advanced colorectal cancer (ACRC): a phase II study of the Gruppo Oncologico dell'Italia Meridionale (GOIM). Ann Oncol. 2009;20(Suppl. 8):viii118 (Abstract L18)

8. Schemper M, Smith TL. A note on quantifying follow-up in studies of failure time. Control Clin Trials. 1996;17:343-346.

9. Grande C, Quintero G, Candamio S, París Bouzas L, Villanueva MJ, Campos B, et al. Biweekly XELOX (capecitabine and oxaliplatin) as first-line treatment in elderly patients with metastatic colorectal cancer. J Geriatr Oncol. 2013;4(2):114-121. 
10. Matsuda C, Honda M, Tanaka C, Fukunaga M, Ishibashi K, Munemoto Y, et al. Multicenter randomized phase II clinical trial of oxaliplatin reintroduction as third- or later-line therapy for metastatic colorectal cancer-biweekly versus standard triweekly XELOX (The ORION Study). Int J Clin Oncol. 2016;21(3):566-572.

11. Matsui T, Nagata N, Hirata K, Okazaki S, Sato S, Nakamura M, et al. Bi-weekly capecitabineoxaliplatin (XELOX) plus bevacizumab as first-line treatment of metastatic colorectal cancer The PHOENiX trial. Anticancer Res. 2016;36(7):3437-3443.

12. Hurwitz H1, Mitchell EP, Cartwright T, Kwok A, Hu S, McKenna E, Patt YZ. A randomized, phase II trial of standard triweekly compared with dose-dense biweekly capecitabine plus oxaliplatin plus bevacizumab as first-line treatment for metastatic colorectal cancer: XELOX-ADVS (Dense Versus Standard). Oncologist. 2012;17(7):937-946. 